\title{
Gravitational waves within the magnetar model of superluminous supernovae and gamma-ray bursts
}

\author{
Wynn C. G. Ho ${ }^{\star}$ \\ Mathematical Sciences, Physics and Astronomy and STAG Research Centre, University of Southampton, Southampton, SO17 1BJ, UK
}

Accepted 2016 August 9. Received 2016 June 17; in original form 2016 May 23

\begin{abstract}
The light curve of many supernovae (SNe) and gamma-ray bursts (GRBs) can be explained by a sustained injection of extra energy from its possible central engine, a rapidly rotating strongly magnetic neutron star (i.e. magnetar). The magnetic dipole radiation power that the magnetar supplies comes at the expense of the star's rotational energy. However, radiation by gravitational waves (GWs) can be more efficient than magnetic dipole radiation because of its stronger dependence on neutron star spin rate $\Omega$, i.e. $\Omega^{6}$ (for a static 'mountain') or $\Omega^{8}$ (for a r-mode fluid oscillation) versus $\Omega^{4}$ for magnetic dipole radiation. Here, we use the magnetic field $B$ and initial spin period $P_{0}$ inferred from SN and GRB observations to obtain simple constraints on the dimensionless amplitude of the mountain of $\varepsilon<0.01$ and r-mode oscillation of $\alpha<1$, the former being similar to that obtained by recent works. We then include $\mathrm{GW}$ emission within the magnetar model. We show that when $\varepsilon>10^{-4}\left(B / 10^{14} \mathrm{G}\right)\left(P_{0} / 1 \mathrm{~ms}\right)$ or $\alpha>0.01\left(B / 10^{14} \mathrm{G}\right)\left(P_{0} / 1 \mathrm{~ms}\right)^{2}$, light curves are strongly affected, with significant decrease in peak luminosity and increase in time to peak luminosity. Thus the GW effects studied here are more pronounced for low $B$ and short $P_{0}$ but are unlikely to be important in modelling SN and GRB light curves since the amplitudes needed for noticeable changes are quite large.
\end{abstract}

Key words: gamma-ray burst: general - gravitational waves - stars: magnetars - stars: neutron - stars: oscillations - supernovae: general.

\section{INTRODUCTION}

A subset of supernovae ( $\mathrm{SNe}$ ) and gamma-ray bursts (GRBs) requires an additional source of energy input over an extended period of time in order to explain the observed evolution of their brightness or light curve. The magnetar model can provide such an energy and timescale (see, e.g. Usov 1992; Dai \& Lu 1998; Zhang \& Mészáros 2001 for GRBs and Maeda et al. 2007; Kasen \& Bildsten 2010; Woosley 2010 for SNe), and this model has been used quite successfully (see, e.g. Rowlinson et al. 2013; Lü et al. 2015 for GRBs and Chatzopoulos et al. 2013; Inserra et al. 2013; Nicholl et al. 2014 for SNe). The magnetar model presupposes that a rapidly rotating magnetar (i.e. neutron star with spin period $P \sim 1 \mathrm{~ms}$ and magnetic field $B \sim 10^{14} \mathrm{G}$; see, e.g. Mereghetti 2008; Mereghetti et al. 2015; Turolla et al. 2015, for review of magnetars) forms during the SN. The newborn magnetar supplies extra energy to power the SN or GRB light curve via its large rotational energy

$E_{\mathrm{rot}}=I \Omega^{2} / 2=2.0 \times 10^{52} \mathrm{erg}(P / 1 \mathrm{~ms})^{-2}$,

where $\Omega(=2 \pi / P)$ and $I\left(\approx 10^{45} \mathrm{~g} \mathrm{~cm}^{2}\right)$ are the magnetar angular spin frequency and moment of inertia, respectively. This rotational

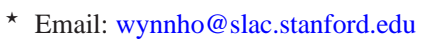

energy is deposited within the SN on the timescale over which the magnetar loses energy via magnetic dipole radiation, i.e.

$t_{\mathrm{mag}}=\left|\frac{E_{\mathrm{rot}}}{\dot{E}_{\mathrm{mag}}}\right|_{\Omega_{0}}=\frac{1}{2 \beta \Omega_{0}^{2}}=2.0 \times 10^{5} \mathrm{~s}_{14}^{-2}\left(P_{0} / 1 \mathrm{~ms}\right)^{2}$,

where $B_{14}=B / 10^{14} \mathrm{G}, \Omega_{0}$ and $P_{0}$ are initial spin frequency and period, respectively, $\beta \equiv B^{2} R^{6} / 6 c^{3} I=6.2 \times 10^{-14} \mathrm{~s} B_{14}^{2}$, and we assume a neutron star mass $M=1.4 M_{\odot}$, radius $R=10 \mathrm{~km}$, and $I=10^{45} \mathrm{~g} \mathrm{~cm}^{2}$ hereafter. Magnetic dipole radiation energy loss $\dot{E}_{\text {mag }}$ is the power supplied by the magnetar $L_{\text {mag }}$ to the $\mathrm{SN}$ and is given by

$$
\begin{aligned}
L_{\mathrm{mag}} & \equiv \dot{E}_{\mathrm{mag}}=-\frac{B^{2} R^{6} \Omega^{4} \sin ^{2} \theta}{6 c^{3}}=-\beta I \Omega^{4} \\
& =-9.6 \times 10^{46} \mathrm{erg} \mathrm{s}^{-1} B_{14}^{2}(P / 1 \mathrm{~ms})^{-4},
\end{aligned}
$$

where $\theta$ is the angle between stellar rotation and magnetic axes (Pacini 1968; Gunn \& Ostriker 1969; see also Spitkovsky 2006; Contopoulos et al. 2014). For simplicity, we assume an orthogonal rotator, i.e. $\sin \theta=1$. It is also worth noting that while the theoretical minimum spin period is $\sim 0.3-0.5 \mathrm{~ms}$ (Cook et al. 1994; Koranda et al. 1997; Haensel et al. 1999), the lowest observed radio pulsar spin period is $1.4 \mathrm{~ms}$ (Hessels et al. 2006).

The millisecond magnetar model for $\mathrm{SNe}$ and GRBs is useful because it provides an easy explanation for the requisite ex- 
tra energy and timescale. These last two map directly to the initial magnetar magnetic field and spin period (see equations 1 and 2), with somewhat weaker dependence on other parameters (see below). However, the effect of emission of gravitational waves (GWs) merits consideration because GW energy loss $\dot{E}_{\mathrm{gw}}$ scales with spin frequency at a higher power than the scaling of magnetic dipole radiation (see equation 1), and thus the assumption and need for rapid rotation can lead to $\dot{E}_{\mathrm{gw}} \gtrsim L_{\mathrm{mag}}$. GWs from a neutron star can be produced in two ways, by a static quadrupolar deformation ('mountain') or by a fluid oscillation (see, e.g. Lasky 2015). For a mountain with size $\varepsilon$, the GW energy loss rate and timescale are (see, e.g. Shapiro \& Teukolsky 1983)

$$
\begin{aligned}
\dot{E}_{\mathrm{gw}} & =-\frac{32}{5} \frac{G I^{2}}{c^{5}} \varepsilon^{2} \Omega^{6}=-\gamma_{\mathrm{e}} I \Omega^{6} \\
& =1.1 \times 10^{46} \mathrm{erg} \mathrm{s}^{-1} \varepsilon_{-4}^{2}(P / 1 \mathrm{~ms})^{-6} \\
t_{\mathrm{gw}} & =\left|\frac{E_{\mathrm{rot}}}{\dot{E}_{\mathrm{gw}}}\right|_{\Omega_{0}}=\frac{1}{2 \gamma_{\mathrm{e}} \Omega_{0}^{4}}=1.8 \times 10^{6} \mathrm{~s}_{-4}^{-2}\left(P_{0} / 1 \mathrm{~ms}\right)^{4},
\end{aligned}
$$

respectively, where $\gamma_{\mathrm{e}} \equiv 32 G I \varepsilon^{2} / 5 c^{5}=1.8 \times 10^{-14} \mathrm{~s}^{3} \varepsilon^{2}$ and $\varepsilon_{-4}=\varepsilon / 10^{-4}$. Alternatively, if the neutron star has a r-mode oscillation with amplitude $\alpha$ (Andersson \& Kokkotas 2001), then the GW energy loss rate and timescale are (see, e.g. Owen et al. 1998)

$$
\begin{aligned}
\dot{E}_{\mathrm{gw}, \mathrm{r}} & \approx-\frac{96 \pi}{15^{2}}\left(\frac{4}{3}\right)^{6} \frac{G M R^{4} \tilde{J}^{2} I}{c^{7} \tilde{I}} \alpha^{2} \Omega^{8}=-\gamma_{\mathrm{r}} I \Omega^{8} \\
& =1.6 \times 10^{46} \mathrm{erg} \mathrm{s}^{-1} \alpha_{-2}^{2}(P / 1 \mathrm{~ms})^{-8} \\
t_{\mathrm{gw}, \mathrm{r}} & =\left|\frac{E_{\mathrm{rot}}}{\dot{E}_{\mathrm{gw}, \mathrm{r}}}\right|_{\Omega_{0}}=\frac{1}{2 \gamma_{\mathrm{r}} \Omega_{0}^{6}}=1.2 \times 10^{6} \mathrm{~s} \alpha_{-2}^{-2}\left(P_{0} / 1 \mathrm{~ms}\right)^{6},
\end{aligned}
$$

respectively, where $\gamma_{\mathrm{r}} \equiv\left(96 \pi / 15^{2}\right)(4 / 3)^{6}\left(G M R^{4} \tilde{J}^{2} / c^{7} \tilde{I}\right) \alpha^{2}=6.6 \times$ $10^{-26} \mathrm{~s}^{5} \alpha^{2}$ and $\alpha_{-2}=\alpha / 10^{-2}$. Note that $\tilde{J}(=0.01635)$ and $\tilde{I}(=$ $\left.I / M R^{2}=0.261\right)$ are derived using a $\Gamma=2$ polytrope with $R=$ $12.53 \mathrm{~km}$ (Owen et al. 1998), whereas we take $R=10 \mathrm{~km}$, but this difference will not change our results qualitatively.

GW emission by the magnetar has a two-fold effect on its ability to provide extra power to a SN or GRB: (1) By causing the neutron star spin rate $\Omega$ to decrease faster than by magnetic dipole radiation (when $t_{\mathrm{gw}}$ or $t_{\mathrm{gw}, \mathrm{r}} \lesssim t_{\mathrm{mag}}$; see below), the timescale over which $E_{\text {rot }}(\Omega)$ is supplied to the SN/GRB is shorter; (2) Since the energy emitted by GWs is not imparted to the SN/GRB, the time evolution of the energy supplied to the SN/GRB changes, and thus the predicted light curve changes. In Section 2, we use (1) and inferred values of initial spin period $P_{0}$ and magnetic field $B$ to obtain the simplest constraint on GW ellipticity $\varepsilon$ and amplitude $\alpha$. In Section 3, we account for GWs within the magnetar model of Kasen \& Bildsten (2010) in order to evaluate the effect on light curves and compare to the magnetar model without GWs. We summarize and briefly discuss our results in Section 4. We note that several recent works performed similar analysis to that done here. Moriya \& Tauris (2016) compare timescales $t_{\mathrm{mag}}$ and $t_{\mathrm{gw}}$ in order to constrain $\varepsilon$ for superluminous $\mathrm{SNe}$, while Lasky \& Glampedakis (2016) do the same for short GRBs; these works assume that the spin period evolution is determined by either magnetic dipole radiation or GW emission due to an ellipticity, as is done in Section 2, whereas their combined effects are considered in Section 3. Kashiyama et al. (2016) use a somewhat more detailed model compared to that presented in Section 3 in order to evaluate effects of GWs (with $\varepsilon$ due to extreme magnetic fields) on light curves of superluminous SNe (see also Zhang \& Mészáros 2001 for GRBs).

\section{MAGNETAR MODEL WITHOUT GWS: GW CONSTRAINTS}

In this section, we consider the simplest GW effect on the magnetar model. We assume that some SN and GRB light curves are well-explained by the magnetar model without GWs. As a result of fits to these light curves, the magnetar initial spin period $P_{0}$ and magnetic field $B$ are extracted (see, e.g. Maeda et al. 2007; Troja et al. 2007; Chatzopoulos et al. 2013; Inserra et al. 2013; Rowlinson et al. 2013; Nicholl et al. 2014; Lü et al. 2015). For the neutron star spin rate to decrease by only magnetic dipole radiation, the timescale for this energy loss must be shorter than that due to GWs, i.e. $t_{\mathrm{mag}}<t_{\mathrm{gw}}$ or

$t_{\mathrm{mag}} / t_{\mathrm{gw}}=\gamma_{\mathrm{e}} \Omega_{0}^{2} / \beta=0.11 B_{14}^{-2} \varepsilon_{-4}^{2}\left(P_{0} / 1 \mathrm{~ms}\right)^{-2}<1$

and $t_{\mathrm{mag}}<t_{\mathrm{gw}, \mathrm{r}}$ or

$t_{\mathrm{mag}} / t_{\mathrm{gw}, \mathrm{r}}=\gamma_{\mathrm{r}} \Omega_{0}^{4} / \beta=0.17 B_{14}^{-2} \alpha_{-2}^{2}\left(P_{0} / 1 \mathrm{~ms}\right)^{-4}<1$.

Thus the constraint on GW ellipticity $\varepsilon$ and r-mode amplitude $\alpha$ are

$$
\begin{aligned}
\varepsilon & <3.0 \times 10^{-4} B_{14}\left(P_{0} / 1 \mathrm{~ms}\right) \\
\alpha & <0.025 B_{14}\left(P_{0} / 1 \mathrm{~ms}\right)^{2},
\end{aligned}
$$

respectively, because otherwise the neutron star spin rate would decrease more quickly than it would in the magnetar model without GWs. Equation (10) is identical to that found in Moriya \& Tauris (2016), and thus our constraints on $\varepsilon$ are the same as theirs. Lasky \& Glampedakis (2016) derive a nearly identical constraint equation for $\varepsilon$ but include an efficiency factor which leads to constraints about ten times smaller. Our constraints on $\varepsilon$ and $\alpha$ are shown in Figs 1 and 2, alongside inferred $P_{0}$ and $B$ compiled by Moriya \& Tauris (2016) for SNe and Lasky et al. (2014) for GRBs; note that these inferred spin period and magnetic field values are derived from fits of observed light curves using a model that assumes no contribution by GW energy loss. We see that ellipticity is constrained to be $\varepsilon<10^{-3}$ and r-mode amplitude is constrained to be $\alpha \lesssim 0.1$ for many SNe. Because of its low inferred magnetic field (thus weaker magnetic dipole radiation loss) and fast inferred rotation rate, ASASSN-15lh has the strongest constraints of $\varepsilon<10^{-4}$ and $\alpha<10^{-2}$. While sources with lower $B$ and/or $P_{0}$ provide stronger $\mathrm{GW}$ constraints, the magnetar contribution to the SN/GRB light curve (i.e. $L_{\text {mag }}$; see equation 3 ) decreases with lower magnetic field, such that shorter spin period is preferred.

\section{MAGNETAR MODEL WITH GRAVITATIONAL WAVES}

Here we build on the magnetar model of Kasen \& Bildsten (2010) (see also Ostriker \& Gunn 1971; Arnett 1979, 1980; Metzger et al. 2015) by accounting for the effect of GW emission by the magnetar (see also Murase et al. 2015; Kashiyama et al. 2016). If the neutron star is a strong enough emitter of GWs, then this energy loss will cause the star's spin frequency to decrease at a faster rate than by pure magnetic dipole radiation. As a consequence, the amount of rotational energy that can be supplied to the SN or GRB is reduced, and the shape of the light curve, e.g. peak luminosity and decay rate, will be altered from that predicted by a model which only considers magnetic dipole radiation.

At early times $\left(t \lesssim t_{\text {diff }}\right.$, where $t_{\text {diff }}$ is the photon diffusion timescale; see below) when the SN or GRB is opaque to photons, the radiated luminosity $L_{\mathrm{rad}}$ (or photon energy loss) is that given by 


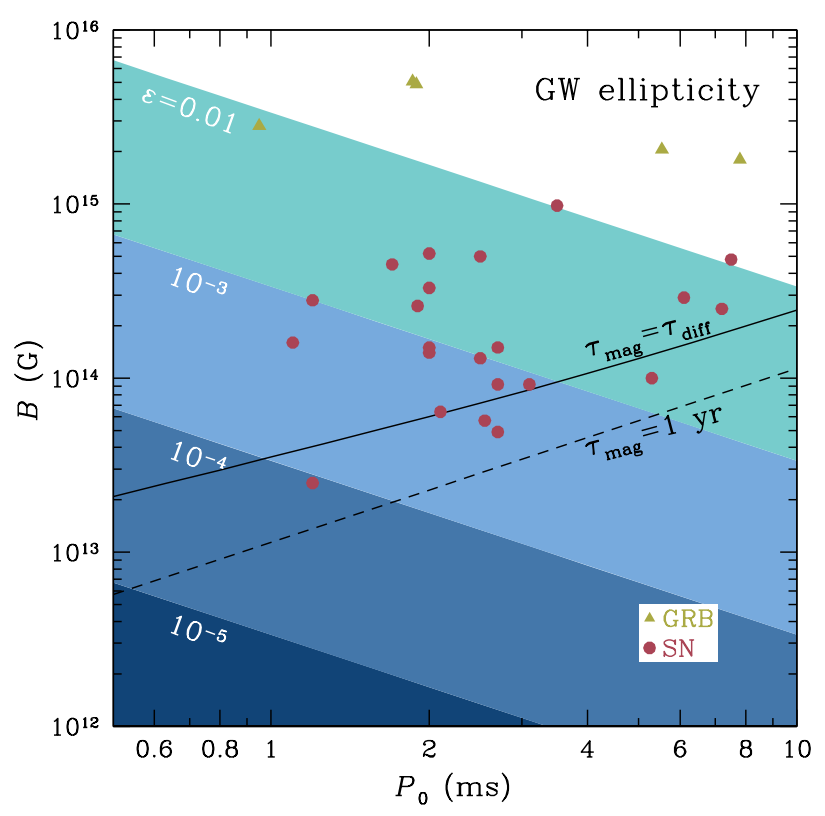

Figure 1. Constraint on GW ellipticity $\varepsilon$ based on equation (10) as a function of neutron star initial spin period $P_{0}$ and magnetic field $B$. Solid line denotes when the magnetic dipole spin-down timescale $t_{\text {mag }}$ (see equation 2) is equal to the radiative diffusion timescale $t_{\mathrm{diff}}$ (see equation 13), and the dashed line denotes when $t_{\text {mag }}$ is equal to 1 yr. Circles are $P_{0}$ and $B$ for $\mathrm{SNe}$ (see Moriya \& Tauris 2016, and references therein) and triangles are $P_{0}$ and $B$ for GRBs (see Lasky et al. 2014, and references therein).

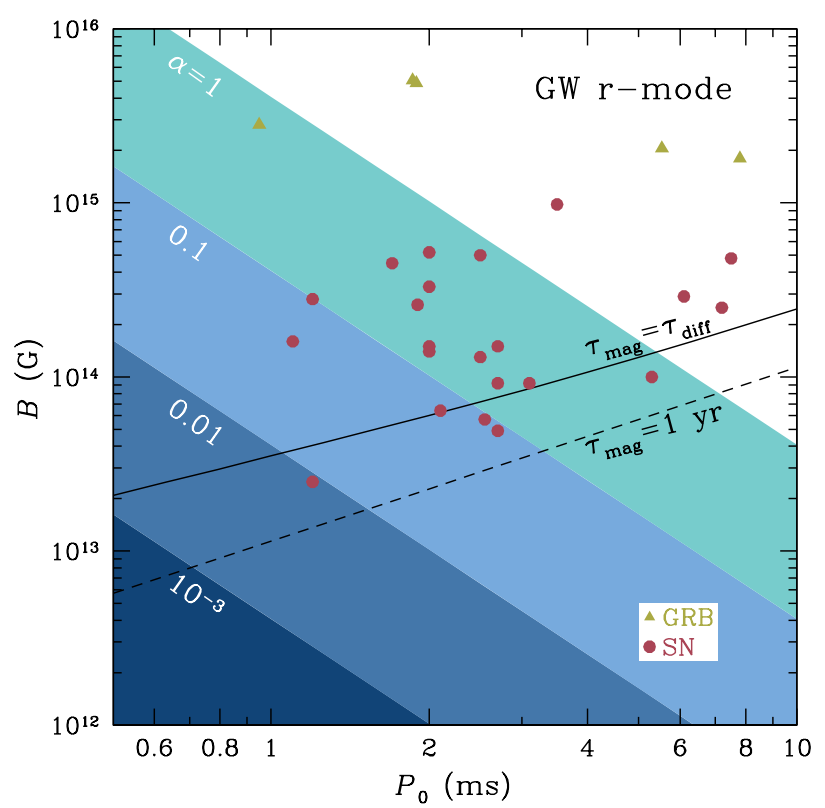

Figure 2. Constraint on GW r-mode amplitude $\alpha$ based on equation (11) as a function of neutron star initial spin period $P_{0}$ and magnetic field $B$. Solid line denotes when the magnetic dipole spin-down timescale $t_{\text {mag }}$ (see equation 2) is equal to the radiative diffusion timescale $t_{\text {diff }}$ (see equation 13), and the dashed line denotes when $t_{\mathrm{mag}}$ is equal to $1 \mathrm{yr}$. Circles are $P_{0}$ and $B$ for SNe (see Moriya \& Tauris 2016, and references therein) and triangles are $P_{0}$ and $B$ for GRBs (see Lasky et al. 2014, and references therein). radiative diffusion, i.e.

$$
\begin{aligned}
L_{\mathrm{rad}} & =4 \pi r^{2}\left[\frac{c}{3 \kappa \rho} \frac{\partial(E / V)}{\partial r}\right] \approx \frac{4 \pi c v}{3 \kappa M_{\mathrm{ej}}} t E=\frac{t E}{t_{\mathrm{diff}}^{2}} \\
& =10^{46} \mathrm{erg} \mathrm{s}^{-1}\left(\frac{t_{\mathrm{diff}}}{10^{6} \mathrm{~s}}\right)^{-2}\left(\frac{E}{10^{52} \mathrm{erg}}\right)\left(\frac{t}{10^{6} \mathrm{~s}}\right),
\end{aligned}
$$

where $E$ is thermal energy, $V$ is volume, $\kappa$ is opacity, $\rho$ is density, $r=v t$, ejecta mass is $M_{\mathrm{ej}}=\rho V$, and expansion velocity is $v \approx$ $\left\{\left[E_{\mathrm{rot}}(\Omega)+E_{\mathrm{SN}}\right] / M_{\mathrm{ej}}\right\}^{1 / 2}$, where $E_{\mathrm{SN}}$ is the initial explosion energy of the SN or GRB. The photon or radiative diffusion timescale is (see, e.g. Arnett 1979)

$$
\begin{aligned}
t_{\mathrm{diff}} & =\left(\frac{3 \kappa M_{\mathrm{ej}}}{4 \pi c v}\right)^{1 / 2}=\left[\frac{3 \kappa M_{\mathrm{ej}}^{3 / 2}}{4 \pi c\left(E_{\mathrm{rot}}+E_{\mathrm{SN}}\right)^{1 / 2}}\right]^{1 / 2} \\
& =2.1 \times 10^{6} \mathrm{~s}\left(\frac{\kappa}{0.2 \mathrm{~cm}^{2} \mathrm{~g}^{-1}}\right)^{1 / 2}\left(\frac{M_{\mathrm{ej}}}{1 M_{\odot}}\right)^{3 / 4}\left(\frac{10^{51} \mathrm{erg}}{E_{\mathrm{rot}}+E_{\mathrm{SN}}}\right)^{1 / 4}(13 .)
\end{aligned}
$$

To determine the light curve or evolution of $L_{\mathrm{rad}}$, we solve the energy equation (see, e.g. Arnett 1979, 1980)

$\frac{\partial E}{\partial t}=-p \frac{\partial V}{\partial t}+L_{\mathrm{mag}}-L_{\mathrm{rad}}$

The first term on the right-hand side is energy lost to expansion due to pressure $p$, the second is energy supplied by the rapidly rotating magnetar, and the third is energy radiated as photons. Note that energy radiated as GWs is accounted for in the lower energy provided by the magnetar. We could also include a term due to heating by Ni decay $L_{\mathrm{Ni}} \sim 10^{43} \mathrm{erg} \mathrm{s}^{-1} e^{-t / 8.8 \mathrm{~d}}$ (Metzger et al. 2015; Kashiyama et al. 2016); however, this would not significantly affect results presented here since we consider rapid rotation, such that $L_{\mathrm{mag}} \gg L_{\mathrm{Ni}}$. When pressure is dominated by radiation, such that $p=(1 / 3) E / V$, then $p(\partial V / \partial t)=E / t$, and equation (14) becomes

$$
\begin{aligned}
\frac{1}{t} \frac{\partial(t E)}{\partial t} & =L_{\mathrm{mag}}-L_{\mathrm{rad}} \\
\frac{\partial(t E)}{\partial t} & =\beta I \Omega^{4} t-\frac{(t E)}{t_{\mathrm{diff}}^{2}(\Omega)} t .
\end{aligned}
$$

Evolution of the neutron star rotation rate is obtained from $\dot{E}_{\mathrm{rot}}=$ $\dot{E}_{\text {mag }}+\dot{E}_{\mathrm{gw}}$, where $\dot{E}_{\text {rot }}=I \Omega \dot{\Omega}=3.9 \times 10^{54} \mathrm{erg} \mathrm{s}^{-1}(P / 1 \mathrm{~ms})^{-3} \dot{P}$, where $\dot{\Omega}$ is time derivative of $\Omega$. For simplicity, we neglect possible accretion on to the newborn neutron star, the effect of which depends on accretion rate and could spin-up or spin-down the star (see, e.g. Piro \& Ott 2011; Melatos \& Priymak 2014). For GWs from an ellipticity $\varepsilon$, the evolution equation for spin frequency is then (see, e.g. Shapiro \& Teukolsky 1983)

$\frac{d \Omega}{d t}=-\beta \Omega^{3}-\gamma_{\mathrm{e}} \Omega^{5}$.

This can be solved analytically to yield (Ostriker \& Gunn 1969)

$t=\int_{\Omega}^{\Omega_{0}} \frac{d \Omega}{\Omega^{3}\left(\beta+\gamma_{\mathrm{e}} \Omega^{2}\right)}=\left[\frac{\gamma_{\mathrm{e}}}{2 \beta^{2}} \ln \left(\frac{\beta+\gamma_{\mathrm{e}} \Omega^{2}}{\Omega^{2}}\right)-\frac{1}{2 \beta \Omega^{2}}\right]_{\Omega}^{\Omega_{0}}$

and, after some algebra,

$\frac{\Omega^{2}}{1-\frac{\gamma_{\mathrm{e}} \Omega^{2}}{\beta} \ln \left(\frac{1+\beta / \gamma_{\mathrm{e}} \Omega^{2}}{1+\beta / \gamma_{\mathrm{e}} \Omega_{0}^{2}}\right)}=\frac{\Omega_{0}^{2}}{1+2 \beta \Omega_{0}^{2} t}$.

Analogous to equation (16), the spin evolution equation for GW from a r-mode is

$\frac{d \Omega}{d t}=-\beta \Omega^{3}-\gamma_{\mathrm{r}} \Omega^{7}$, 


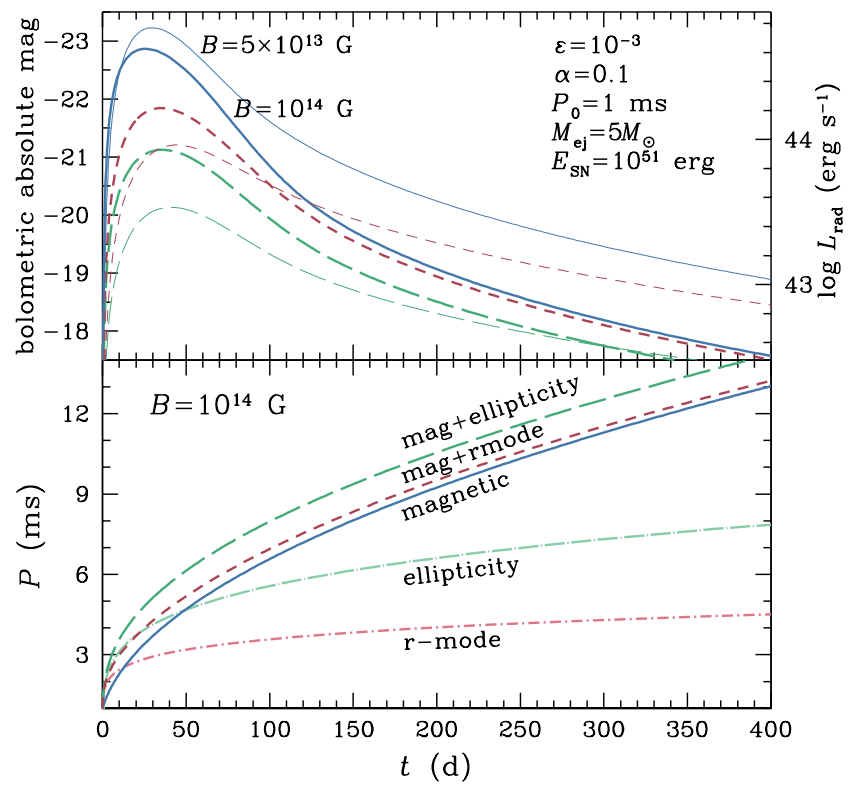

Figure 3. Light curves of radiated luminosity $L_{\mathrm{rad}}$ and spin period $P$ as a function of time. Solid lines are for a model which only includes magnetic dipole energy loss $L_{\mathrm{mag}}$, with parameters $P_{0}=1 \mathrm{~ms}, M_{\mathrm{ej}}=5 M_{\odot}, E_{\mathrm{SN}}=$ $10^{51} \mathrm{erg}$, and either $B=10^{14} \mathrm{G}$ (bold lines) or $B=5 \times 10^{13} \mathrm{G}$ (light lines). Long-dashed lines are for a model which includes magnetic dipole and GW energy loss $\dot{E}_{\mathrm{gW}}$, with ellipticity $\varepsilon=10^{-3}$. Short-dashed lines are for a model which includes magnetic dipole and GW energy loss $\dot{E}_{\mathrm{gw}, \mathrm{r}}$, with rmode amplitude $\alpha=0.1$. Dot-dashed lines are for spin period evolution by only GW ellipticity or r-mode.

with a solution that is given by

$$
\frac{\Omega^{2}}{1-\left(\frac{\gamma_{\mathrm{r}} \Omega^{4}}{\beta}\right)^{1 / 2} \tan ^{-1}\left[\frac{\left(\gamma_{\mathrm{r}} \Omega_{0}^{4} / \beta\right)^{1 / 2}\left(1-\Omega^{2} / \Omega_{0}^{2}\right)}{1+\gamma \Omega_{0}^{2} \Omega^{2} / \beta}\right]}=\frac{\Omega_{0}^{2}}{1+2 \beta \Omega_{0}^{2} t} .
$$

For equations (18) and (20), it is clear that when there is no additional torque (such as that due to GWs), i.e. $\gamma_{\mathrm{e}}=0$ and $\gamma_{\mathrm{r}}=0$, the spin frequency evolution $\Omega(t)$ reduces to that due to a magnetic dipole $\Omega(t)=\Omega_{0}\left(1+2 \beta \Omega_{0}^{2} t\right)^{-1 / 2}=\Omega_{0}(1+$ $\left.t / t_{\mathrm{mag}}\right)^{-1 / 2}$ and substituting into equation (3) yields $L_{\mathrm{mag}}(t)=-9.6 \times$ $10^{46} \mathrm{erg} \mathrm{s}^{-1} B_{14}^{2}\left(P_{0} / 1 \mathrm{~ms}\right)^{-4}\left(1+t / t_{\mathrm{mag}}\right)^{-2}$.

Examples of solutions of the coupled ${ }^{1}$ evolution equations (15) and (16) with $\varepsilon=10^{-3}$ or equations (15) and (19) with $\alpha=0.1$ are shown in Figs 3 and 4. Initial spin period is $P_{0}=1 \mathrm{~ms}$ for Fig. 3 and $2 \mathrm{~ms}$ for Fig. 4. Results for two magnetic fields $\left(B=5 \times 10^{13}\right.$ and $\left.10^{14} \mathrm{G}\right)$ are shown in Fig. 3, while two ejecta masses $\left(M_{\mathrm{ej}}=M_{\odot}\right.$ and $\left.5 M_{\odot}\right)$ are shown in Fig. 4. Bottom panels also show spin period evolution due to only a GW ellipticity or r-mode, i.e. $P=P_{0}\left(1+2 t / t_{\mathrm{gw}}\right)^{1 / 4}$ or $P=P_{0}\left(1+3 t / t_{\mathrm{gw}, \mathrm{r}}\right)^{1 / 6}$, respectively, compared to $P=P_{0}\left(1+t / t_{\mathrm{mag}}\right)^{1 / 2}$ for only magnetic dipole radiation (see after equation 20). GW emission determines the spin evolution at early times ( $t \lesssim$ several days), while magnetic dipole radiation dominates at late times so that $L_{\mathrm{mag}} \propto\left(1+t / t_{\mathrm{mag}}\right)^{-2}$ (see after equation 20).

It is clear that GW energy loss causes the neutron star to increase its spin period more quickly at early times, and this de-

1 It is simple to solve either differential equations for $\Omega$ rather than use the analytic solution of $\Omega(t)$ given by equations (18) or (20).

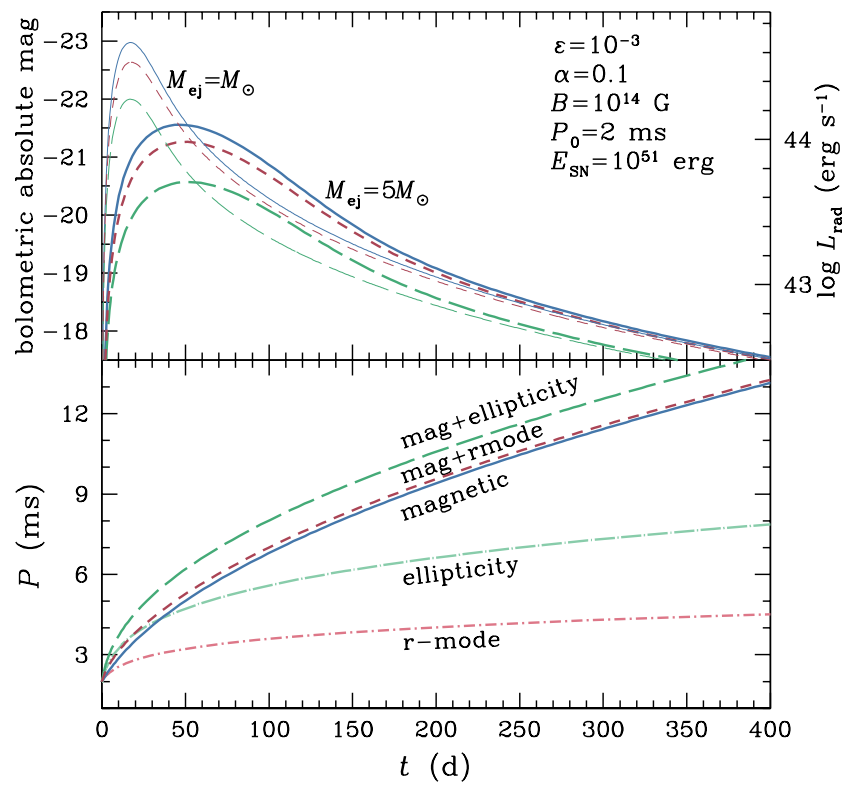

Figure 4. Light curves of radiated luminosity $L_{\mathrm{rad}}$ and spin period $P$ as a function of time. Solid lines are for a model which only includes magnetic dipole energy loss $L_{\mathrm{mag}}$, with parameters $B=10^{14} \mathrm{G}, P_{0}=2 \mathrm{~ms}, E_{\mathrm{SN}}=$ $10^{51} \mathrm{erg}$, and either $M_{\mathrm{ej}}=5 M_{\odot}$ (bold lines) or $M_{\mathrm{ej}}=M_{\odot}$ (light lines). Long-dashed lines are for a model which includes magnetic dipole and GW energy loss $\dot{E}_{\mathrm{gw}}$, with ellipticity $\varepsilon=10^{-3}$. Short-dashed lines are for a model which includes magnetic dipole and GW energy loss $\dot{E}_{\mathrm{gw}, \mathrm{r}}$, with rmode amplitude $\alpha=0.1$ Dot-dashed lines are for spin period evolution by only GW ellipticity or r-mode.

creases the amount of rotational energy that can be used to power the SN/GRB light curve. For lower magnetic fields, the effect of GWs is more dramatic, which can be understood simply from the ratio of timescales given by equations (8) and (9). Some effects of GWs are illustrated in Figs 5 and 6, which plots peak photon luminosity $L_{\text {peak }}$ ( $\equiv$ maximum $L_{\mathrm{rad}}$ ) and time to peak luminosity $t_{\text {peak }} \equiv t\left(L_{\text {peak }}\right)$. The different lines of $L_{\text {peak }}\left(t_{\text {peak }}\right)$ are calculated assuming either constant magnetic field $B$ and varying initial spin period $P_{0}$ or constant $P_{0}$ and varying $B$. Similar plots are shown in Kasen \& Bildsten (2010) for the case of magnetic dipole radiation only, and our results for this case are comparable. Quantitative differences are due to a factor of two in the magnetic dipole radiation timescale $t_{\text {mag }}$ (see equation 2), where Kasen \& Bildsten (2010) assume $\sin ^{2} \theta=1 / 2$ (see equation 3 ) and a factor of two and $E_{\mathrm{rot}}\left(\Omega_{0}\right)$ in expansion velocity and hence $t_{\text {diff }}$ (see equation 13). We also denote the radiative diffusion timescale $t_{\mathrm{diff}}(\leqslant 82 \mathrm{~d}$ for the parameters used here; see equation 13) by the shaded region in Figs 5 and 6.

For higher magnetic field strengths, magnetic dipole radiation is stronger, and peak luminosities and times are the same when neglecting or including the effect of GWs. For the cases shown here $\left(\varepsilon=10^{-3}\right.$ or $\left.\alpha=0.1\right), t_{\text {peak }}$ shifts to later times and $L_{\text {peak }}$ is lower starting at $B \sim 3 \times 10^{14} \mathrm{G}\left(1 \mathrm{~ms} / P_{0}\right)$ for a GW ellipticity and $B \sim 4 \times 10^{14} \mathrm{G}\left(1 \mathrm{~ms} / P_{0}\right)^{2}$ for a $\mathrm{GW}$ r-mode (see equations 8 and 9 , respectively). These effects become significant at $B \lesssim 10^{14} \mathrm{G}$, and there is no longer a one-to-one mapping between $P_{0}-B$ and $t_{\text {peak }}-L_{\text {peak }}$. 


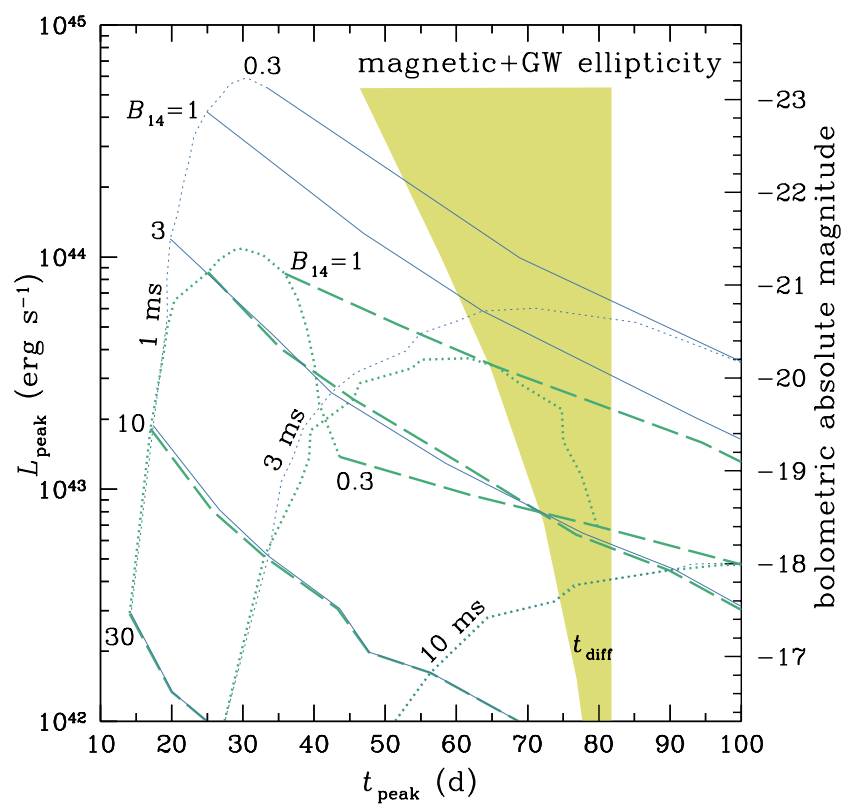

Figure 5. Parameter space of peak radiated photon luminosity $L_{\text {peak }}$ and time to peak luminosity $t_{\text {peak }}$. Light solid lines are for a model which only includes magnetic dipole energy loss, where magnetic field $B=10^{14} B_{14}$ (labelled) is held constant and initial spin period $P_{0}$ is varied, while light dotted lines are for constant $P_{0}$ (labelled) and varying $B$. Heavy dashed and dotted lines are the same but for a model which includes magnetic dipole and GW energy loss, with ellipticity $\varepsilon=10^{-3}$. Shaded region shows range of diffusion time which varies because $t_{\text {diff }}=t_{\text {diff }}\left[E_{\text {rot }}\left(\Omega_{\text {peak }}\right)\right]$. All models shown assume $E_{\mathrm{SN}}=10^{51} \mathrm{erg}$ and $M_{\mathrm{ej}}=5 M_{\odot}$.

\section{DISCUSSION}

The theoretical model of converting rotational energy of a rapidly rotating magnetar into magnetic dipole energy and this energy then powering ejecta of SNe and GRBs has been successful in matching the observed light curve of SNe and GRBs. Here we consider the possible effects of GW emission on this magnetar model, especially in light of the recent detection of GWs (Abbott et al. 2016a). We use the magnetar model to obtain simple constraints on the neutron star ellipticity $\varepsilon$ (see also Lasky \& Glampedakis 2016; Moriya \& Tauris 2016) and r-mode oscillation amplitude $\alpha$, since large values of $\varepsilon$ or $\alpha$ would cause a rapidly rotating star to emit GWs and lose rotational energy at a faster rate than by magnetic dipole radiation (see also Dai et al. 2016). We then account for GW emission processes within the magnetar model and show how the evolution of the spin period and photon luminosity changes as a result of inclusion of GW emission (see also Kashiyama et al. 2016 for the case of $\varepsilon$ ).

Our constraint of $\varepsilon<0.01$ from Section 2 and assumed value of $\varepsilon=10^{-3}$ in Section 3 are relatively large in magnitude. GW detectors have thus far not detected GWs from rotating neutron stars, with upper limits of $\varepsilon \sim 10^{-3}$ (Aasi et al. 2015, 2016) and $\varepsilon \sim 10^{-6}-10^{-4}$ (Aasi et al. 2015; Abbott et al. 2016b) for different frequency regimes (see also Aasi et al. 2014). Note that the neutron stars examined are much older than the magnetars considered in the present work. From a theoretical perspective, elastic deformations have maximum $\varepsilon \sim 10^{-5}$ for neutron stars and $\sim 10^{-3}$ for more exotic stars (Pitkin 2011; Johnson-McDaniel \& Owen 2013). Ellipticities created by strong magnetic fields can have $\varepsilon \approx 10^{-6}$ (at a toroidal field strength of $10^{15} \mathrm{G}$ and increasing as $B^{2}$ or de-

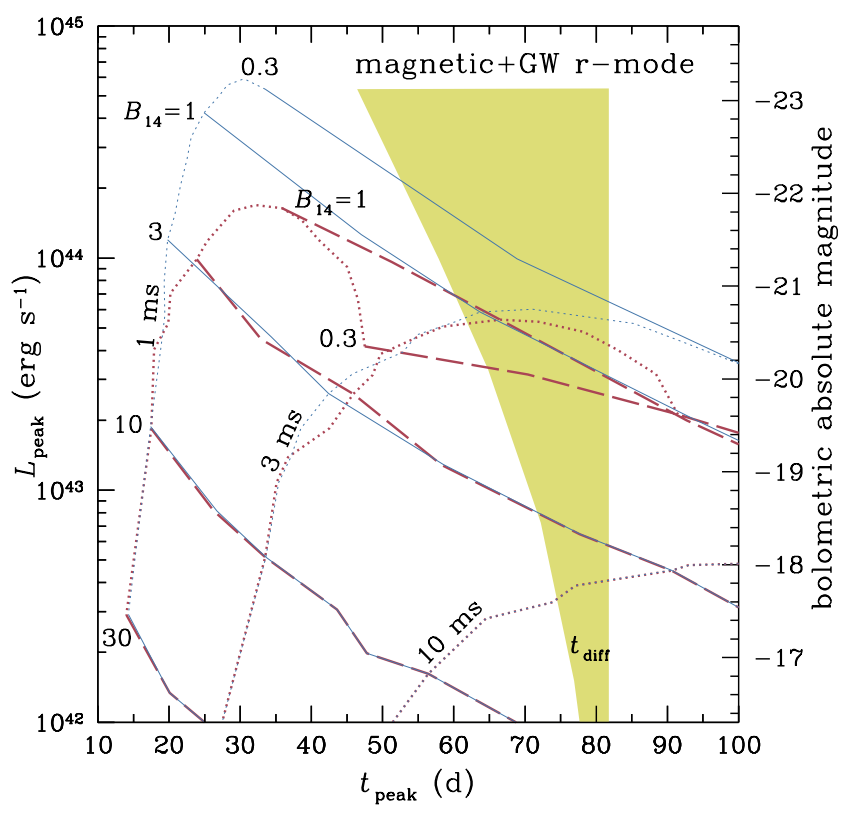

Figure 6. Parameter space of peak radiated photon luminosity $L_{\text {peak }}$ and time to peak luminosity $t_{\text {peak }}$. Light solid lines are for a model which only includes magnetic dipole energy loss, where magnetic field $B=10^{14} B_{14}$ (labelled) is held constant and initial spin period $P_{0}$ is varied, while light dotted lines are for constant $P_{0}$ (labelled) and varying $B$. Heavy dashed and dotted lines are the same but for a model which includes magnetic dipole and GW energy loss, with r-mode amplitude $\alpha=0.1$. Shaded region shows range of diffusion time which varies because $t_{\text {diff }}=t_{\text {diff }}\left[E_{\text {rot }}\left(\Omega_{\text {peak }}\right)\right]$. All models shown assume $E_{\mathrm{SN}}=10^{51} \mathrm{erg}$ and $M_{\mathrm{ej}}=5 M_{\odot}$.

creasing as $B$; Cutler 2002) to $\varepsilon \sim 10^{-3}$ (Melatos \& Priymak 2014; Lasky \& Glampedakis 2016). However the optimal geometry for strong GW emission, i.e. orthogonal magnetic and rotation axes, may not occur even in the presence of extreme magnetic fields (see Lasky \& Glampedakis 2016, for discussion; see also Lai 2001).

Similarly to $\varepsilon$, the constraint of $\alpha<1$ from Section 2 and assumed value of $\alpha=0.1$ in Section 3 are large amplitudes. GW detectors have set upper limits in a wide range of $\alpha \sim 10^{-5}-$ 0.1 , depending on frequency (Aasi et al. 2015). X-ray observations yield upper limits of $\sim 10^{-6}$ (Mahmoodifar \& Strohmayer 2013), as well as possible detection of r-modes with amplitude $\alpha \sim 10^{-5}-10^{-3}$ in two neutron stars (Strohmayer \& Mahmoodifar 2014a,b; Lee 2014), although the observed spin behaviour of one of these stars suggests surface phenomena which would not generate GWs or impact the stellar spin rate (Andersson et al. 2014). But again these constraints are derived for much older neutron stars. Note that we neglect evolution of the r-mode amplitude since $\alpha$ can reach saturation at $10^{-3}$ or much lower (Arras et al. 2003; Bondarescu \& Wasserman 2013) in $\lesssim 10^{3}$ s (Owen et al. 1998; Alford \& Schwenzer 2014) at $B \leqslant 10^{15} \mathrm{G}$ (Ho \& Lai 2000) and thus its evolution is not relevant for $\mathrm{SNe}$ but may be relevant for GRBs (Yu et al. 2010; Cheng \& Yu 2014). However it is important to keep in mind that there is great uncertainty in our understanding of the physics of r-modes (see Ho et al. 2011; Haskell et al. 2012, for discussion).

Finally, even with a large ellipticity, GWs produced by a newborn rapidly rotating magnetar would be difficult to detect, unless the source is particularly nearby (see Kashiyama et al. 2016 for SNe and Lasky \& Glampedakis 2016 for GRBs). The same is true 
for a large amplitude r-mode oscillation, where the GW strain is $h \sim 10^{-24}(10 \mathrm{Mpc} / d)$ and $d$ is source distance.

\section{ACKNOWLEDGEMENTS}

WCGH thanks Nils Andersson for discussion and Bob Nichol, Szymon Prajs, and the anonymous referee for helpful comments. WCGH acknowledges support from the Science and Technology Facilities Council (STFC) in the United Kingdom.

\section{REFERENCES}

Aasi J. et al., 2014, ApJ, 785, 119

Aasi J. et al., 2015, ApJ, 813, 39

Aasi J. et al., 2016, Phys. Rev. D, 93, 042007

Abbott B. P. et al., 2016a, Phys. Rev. Lett., 116, 061102

Abbott B. P. et al., 2016b, Phys. Rev. D, 94, 042002

Alford M. G., Schwenzer K., 2014, ApJ, 781, 26

Andersson N., Kokkotas K. D., 2001, Int. J. Mod. Phys., 10, 381

Andersson N., Jones D. I., Ho W. C. G., 2014, MNRAS, 442, 1786

Arnett W. D., 1979, ApJ, 230, L37

Arnett W. D., 1980, ApJ, 237, 541

Arras P., Flanagan E. E., Morsink S. M., Schenk A. K., Teukolsky S. A., Wasserman I., 2003, ApJ, 591, 1191

Bondarescu R., Wasserman I., 2013, ApJ, 778, 9

Chatzopoulos E., Wheeler J. C., Vinko J., Horvath Z. L., Nage A., 2013, ApJ, 773,76

Cheng Q., Yu Y.-W., 2014, ApJ, 786, L13

Contopoulos I., Kalapotharakos C., Kazanas D., 2014, ApJ, 781, 46

Cook G. B., Shapiro S. L., Teukolsky S. A., 1994, ApJ, 423, L117

Cutler C., 2002, Phys. Rev. D, 66, 084025

Dai Z. G., Lu T., 1998, A\&A, 333, L87

Dai Z. G., Wang S. Q., Wang J. S., Wang L. J., Yu Y. W., 2016, ApJ, 817, 132

Gunn J. E., Ostriker J. P., 1969, Nature, 221, 454

Haensel P., Lasota J. P., Zdunik J. L., 1999, A\&A, 344, 151

Haskell B., Degenaar N., Ho W. C. G., 2012, MNRAS, 424, 93

Hessels J. W. T., Ransom S. M., Stairs I. H. , Freire P. C. C., Kaspi V. M., Camilo F., 2006, Science, 311, 1901

Ho W. C. G., Lai D., 2000, ApJ, 543, 386

Ho W. C. G., Andersson N., Haskell B., 2011, Phys. Rev. Lett., 107, 101101 Inserra C. et al. 2013, ApJ, 770, 128

Johnson-McDaniel N. K., Owen B. J., 2013, Phys. Rev. D, 88, 044004

Kasen D., Bildsten L, 2010, ApJ, 717, 245

Kashiyama K., Murase K., Bartos I., Kiuchi K., Margutti R., 2016, ApJ, 818,94

Koranda S., Stergioulas N., Friedman J. L., 1997, ApJ, 488, 799

Lai D., in Centrella J. M., ed., AIP Conf. Proc. 575, Astrophysical Sources for Ground-Based Gravitational Wave Detectors. Am. Inst. Phys., New York, p. 246

Lasky P. D., 2015, Publ. Astron. Soc. Australia, 32, e034

Lasky P. D., Glampedakis K., 2016, MNRAS, 458, 1660

Lasky P. D., Haskell B., Ravi V., Howell E. J., Coward D. M., 2014, Phys. Rev. D, 89, 047302

Lee U., 2014, MNRAS, 442, 3037

Lü H.-J., Zhang B., Lei W.-H., Lasky P. D., 2015, ApJ, 805, 89

Maeda K. et al., 2007, ApJ, 666, 1069

Mahmoodifar S., Strohmayer T., 2013, ApJ, 773, 140

Melatos A., Priymak M. 2014, ApJ, 794, 170

Mereghetti S., 2008, A\&A Rev., 15, 225

Mereghetti S., Pons J. A., Melatos A., 2015, Space Sci. Rev., 191, 315

Metzger B. D., Margalit B., Kasen D., Quataert E., 2015, MNRAS, 454, 3311

Moriya T. J., Tauris T. M., 2016, MNRAS, 460, L55

Murase K., Kashiyama K., Kiuchi K., Bartos I., 2015, ApJ, 805, 82
Nicholl M. et al., 2014, MNRAS, 444, 2096

Ostriker J. P., Gunn J. E., 1969, ApJ, 157, 1395

Ostriker J. P., Gunn J. E., 1971, ApJ, 164, L95

Owen B. J., Lindblom L., Cutler C., Schutz B. F., Vecchio A., Andersson, N., 1998, Phys. Rev. D, 58, 084020

Pacini F., 1968, Nature, 219, 145

Piro A. L., Ott C. D., 2011, ApJ, 736, 108

Pitkin M., 2011, MNRAS, 415, 1849

Rowlinson A., O'Brien P. T., Metzger B. D., Tanvir N. R., Levan A. J., 2013, MNRAS, 430, 1061

Shapiro S. L., Teukolsky S. A., 1983, Black Holes, White Dwarfs, and Neutron Stars. Wiley, New York

Spitkovsky A., 2006, ApJ, 648, L51

Strohmayer T., Mahmoodifar S., 2014a, ApJ, 784, 72

Strohmayer T., Mahmoodifar S., 2014b, ApJ, 793, L38

Troja E. et al. 2007, ApJ, 665, 599

Turolla R., Zane S., Watts A. L., 2015, Rep. Prog. Phys., 78, 116901

Usov V. V., 1992, Nature, 357, 427

Woosley S. E., 2010, ApJ, 719, L204

Yu Y.-W., Cheng K. S., Cao X.-F., 2010, ApJ, 715, 477

Zhang B., Mészáros P., 2001, ApJ, 552, L35 